\title{
Funcionamiento de una membrana de zeolita 4-A comercial en la deshidratación de disolventes industriales mediante pervaporación
}

\author{
A.M. URTIAGA, E.D. GORRI, C. CASADO, I. ORTIZ \\ Departamento de Ingeniería Química. ETS Ingenieros Industriales y de Telecomunicación. Universidad de Cantabria. Santander. España.
}

\begin{abstract}
En este trabajo se estudia el comportamiento de una membrana comercial de pervaporación con una capa activa de zeolita NaA (Smart Chem., Reino Unido) aplicada a la separación de dos mezclas de origen industrial: tetrahidrofurano/agua con un contenido inicial de agua de 7,9\% en peso y acetona/ agua con un contenido inicial de agua de 3,25\% en peso. Se ha caracterizado el flujo de agua y la selectividad de la separación, estudiándose la influencia de la composición y de la temperatura, en el rango $40{ }^{\circ} \mathrm{C}-65{ }^{\circ} \mathrm{C}$ sobre ambos factores. Asimismo, se ha estudiado la estabilidad de la membrana en ciclos consecutivos de operación.
\end{abstract}

Palabras clave: membrana zeolita NaA, pervaporación, tetrahidrofurano, acetona, deshidratación, estabilidad.

\section{Behavior of a zeolite 4-A commercial membrane in the dehydration of industrial solvents by pervaporation}

The performance of a commercial zeolite NaA pervaporation membrane supplied by Smart Chem., U.K.) was studied with respect to the dehydration of industrial mixtures of tetrahydrofurane/water $\left(\mathrm{C}_{\mathrm{H} 2 \mathrm{O}} \leq 7.9 \mathrm{wt} . \%\right)$ and acetone/water $\left(\mathrm{C}_{\mathrm{H} 2 \mathrm{O}} \leq 3.25 \mathrm{wt} . \%\right)$. The influence of water concentration and temperature in the range $40{ }^{\circ} \mathrm{C}-65^{\circ} \mathrm{C}$ on fluxes and selectivities was described. The stability of the membrane in consecutive operation cycles is also reported.

Keywords: Zeolite membrane, pervaporation, tetrahydrofuran, acetone, dehydration, stability

\section{INTRODUCCIÓN}

La aplicación de materiales cerámicos en tecnologías de separación está siendo impulsada por el desarrollo de nuevas membranas y nuevos procesos. En el caso de la pervaporación los avances más recientes se encuentran en el desarrollo de dos tipos de membranas cerámicas: i) membranas de zeolitas $(1,2)$ y ii) membranas de sílice amorfa $(3,4)$, con un tamaño de poro en la capa selectiva en el rango de $0,4 \mathrm{~nm}$ a $0,5 \mathrm{~nm}$. Este tipo de membranas se adapta al problema de separación planteado como la deshidratación de disolventes orgánicos de uso frecuente en la síntesis de productos químicos y farmacéuticos, especialmente en los disolventes que forman azeótropos con agua, una característica que dificulta e incluso hace económicamente inviable la separación de las mezclas mediante las habituales operaciones de destilación. Otras ventajas de las membranas cerámicas respecto a las poliméricas son su elevada estabilidad térmica y mecánica. La aparición en fechas recientes de membranas cerámicas comerciales permite prever el desarrollo de esta tecnología.

En el proceso de pervaporación aplicado a la separación de una mezcla líquida totalmente miscible de agua en un disolvente orgánico, el agua se adsorbe preferentemente en la membrana hidrófila y difunde a través de los poros, para evaporarse en la otra cara de la membrana, que se mantiene en condiciones de baja presión.

En este trabajo se presentan los resultados obtenidos en el estudio del comportamiento de una membrana comercial con una capa activa de zeolita NaA aplicada a la separación de dos mezclas de origen industrial: tetrahidrofurano/agua y acetona/agua. Los problemas asociados a la separación de estas mezclas mediante la operación habitual de destilación son que la mezcla THF / agua con un 95\% en peso de THF forma un azeótropo a presión atmosférica. En el caso de la mezcla acetona/ agua las composiciones de las fases líquida y vapor en equilibrio son muy similares para contenidos de agua inferiores a 3,5\% en peso (5).

El funcionamiento de la membrana se ha caracterizado en términos de flujo de los componentes individuales de la mezcla y de la selectividad de la separación, estudiándose la influencia de la composición y de la temperatura sobre ambos factores. Asimismo, se ha estudiado la estabilidad de la membrana en ciclos consecutivos de operación.

\section{METODOLOGÍA EXPERIMENTAL}

\subsection{Membranas y materiales}

Las membranas hidrófilas utilizadas para esta investigación son membranas comerciales compuestas con una capa activa de zeolita $\mathrm{NaA}$, fabricadas por Smart Chemical Co. Ltd. (Reino Unido). Las membranas básicamente están hechas de una capa activa de zeolita $\mathrm{NaA}$, depositada sobre la cara interna de un soporte cerámico poroso con geometría cilíndrica. El módulo de membranas posee las siguientes características: (1) área efectiva: 0,05 m², (2) espesor de la capa de membrana selectiva: 5-10 $\mu \mathrm{m}$, (3) diámetro de poros: 0,42 nm, (4) rango de $\mathrm{pH}$ recomendado: 6-8, (5) máxima temperatura de operación: $150^{\circ} \mathrm{C}$, (6) presión máxima: 10 bar, (7) sensibilidad de iones metálicos divalentes $>1 \%$. El uso de esta membrana comercial para la deshidratación de mezclas t-butanol/agua ha sido publicado recientemente por Gallego-Lizón et al. (6).

Se trabajó en la separación de dos mezclas industriales: Mezcla 1: tetrahidrofurano/agua con un contenido inicial de agua de 7,9\% en peso; Mezcla 2: acetona/agua con un contenido inicial de agua de $3,25 \%$ en peso. 


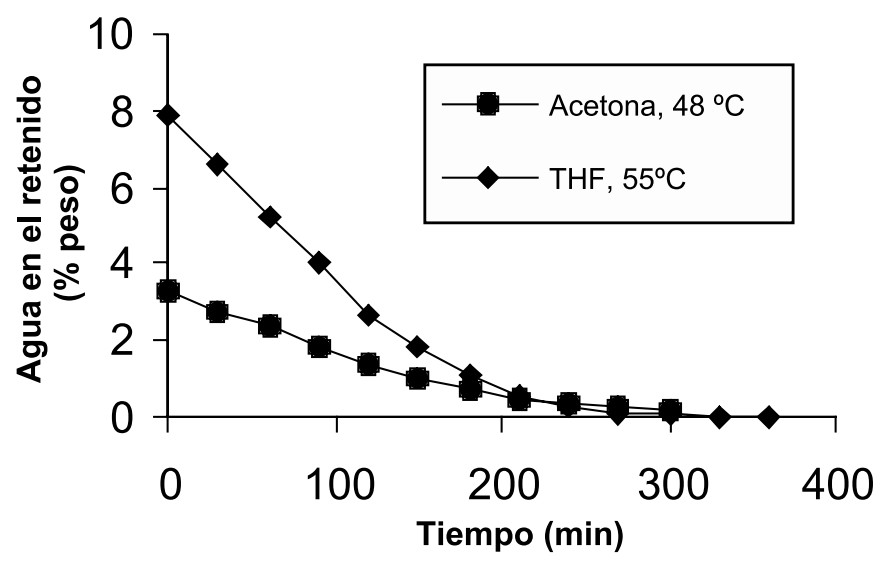

Figura 1. Variación de la concentración de agua en el tanque de alimentación frente a tiempo de experimentación

\subsection{Equipos y procedimientos}

Los experimentos de pervaporación se llevaron a cabo en una instalación a escala de laboratorio, utilizada por los autores en otros estudios (7-9), donde fue montado el módulo tubular de membranas zeolíticas. La mezcla líquida de alimentación (vol. = 2 l) se colocó en un tanque termostatizado a la temperatura de proceso y se hizo recircular a través de la instalación por medio de una bomba centrífuga. El vacío en la zona de permeado se mantuvo por debajo de 20 mbar. Se extrajeron muestras de retenido y de permeado a intervalos regulares de tiempo. Los vapores de permeado fueron condensados por medio de dos trampas frías colocadas en paralelo y enfriadas con nitrógeno líquido. La concentración de agua en el retenido fue determinada por titulación utilizando el método de Karl Fischer. La concentración de THF en el permeado se determinó mediante medida del índice de refracción.

\section{RESULTADOS Y DISCUSIÓN}

La figura 1 muestra la evolución con el tiempo de la concentración de agua en THF, ensayo realizado a $55^{\circ} \mathrm{C}$ y en acetona, ensayo realizado a $48^{\circ} \mathrm{C}$. La concentración de agua en THF se rebaja desde el 7,9\% inicial hasta valores por debajo del $0,1 \%$ en peso. En el caso de la acetona, desde el 3,25\% inicial al 0,2\% final. Se concluye que la tecnología permite deshidratar el THF y la acetona hasta un grado de sequedad suficiente para la recirculación del disolvente al proceso industrial.

La figura 2 muestra la dependencia del flujo con la concentración de agua en el retenido, en función de la temperatura de operación. Se observa que un incremento de la temperatura hace que aumente el flujo de agua a través de la membrana. Para un $7 \%$ de agua en THF el flujo aumenta desde $0,6 \mathrm{~kg} / \mathrm{m}^{2}$-h obtenido a $45^{\circ} \mathrm{C}$ a $1,5 \mathrm{~kg} / \mathrm{m}^{2}-\mathrm{h}$ obtenido a $65^{\circ} \mathrm{C}$. Estos valores de flujo de agua son superiores a los reportados en la bibliografía (10) con membranas zeolitas tipo A, de 0,49 kg/ $\mathrm{m}^{2}-\mathrm{h}$ para mezclas $95 \% \mathrm{THF} / 5 \%$ agua a $60{ }^{\circ} \mathrm{C}$. Para un $3 \%$ de agua en acetona el flujo de agua tiene un valor de $0.13 \mathrm{~kg} / \mathrm{m}^{2} \mathrm{~h}$ a $40^{\circ} \mathrm{C}$ y de 0.314 $\mathrm{kg} / \mathrm{m}^{2} \mathrm{~h}$ a $48^{\circ} \mathrm{C}$. Este valor es inferior al reportado en la bibliografía (2), de $0,83 \mathrm{~kg} / \mathrm{m}^{2}-\mathrm{h}$, para una mezcla $95 \%$ acetona $/ 5 \%$ agua a $50{ }^{\circ} \mathrm{C}$.

Los resultados que se muestran a continuación cuestionan la estabilidad de la membrana en las condiciones de proceso. La figura 3 muestra los resultados obtenidos en 9 ciclos consecutivos de deshidratación de THF realizados a $55{ }^{\circ} \mathrm{C}$; por razones de claridad, en la figura 3(b) solo se muestran 4 ciclos representativos de las tendencias.
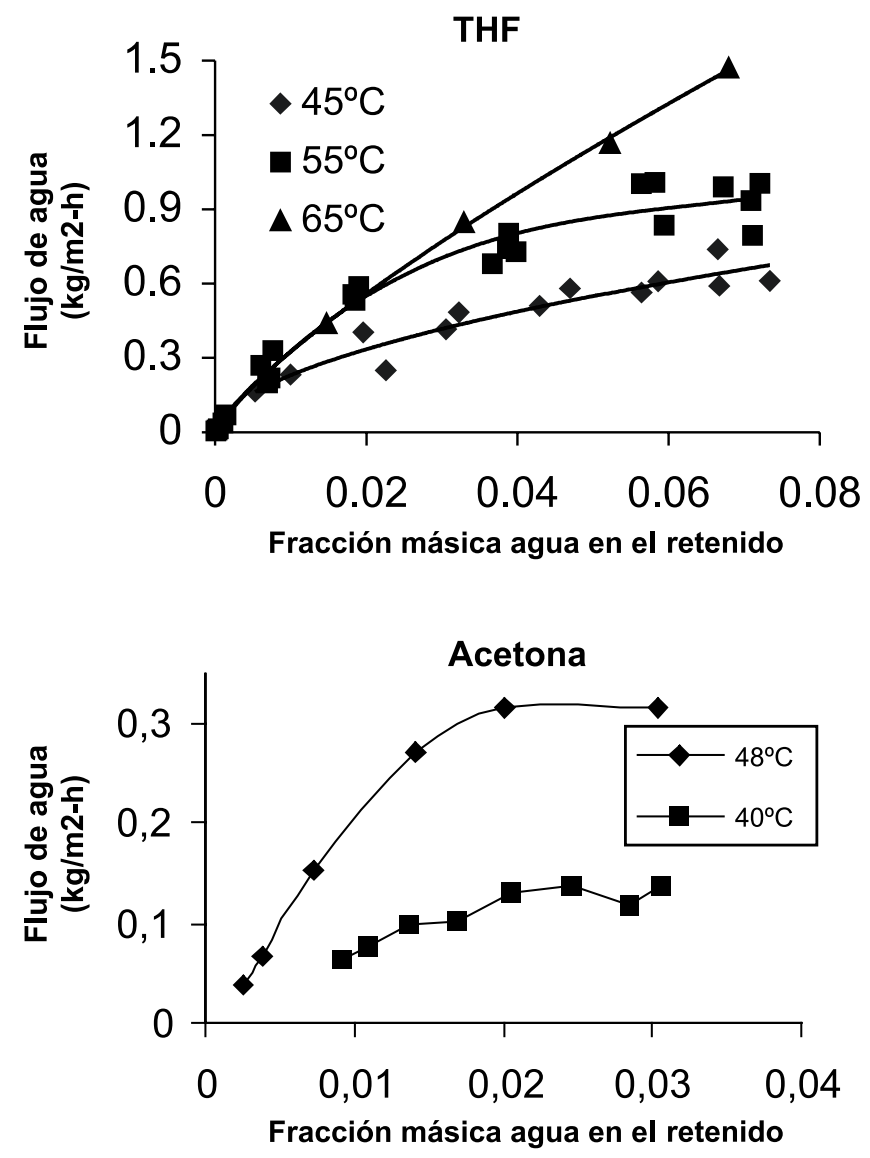

Figura 2. Flujo de agua en función de la fracción másica de agua en el retenido. Influencia de la temperatura. (a) mezcla THF/agua. (b) mezcla acetona/agua

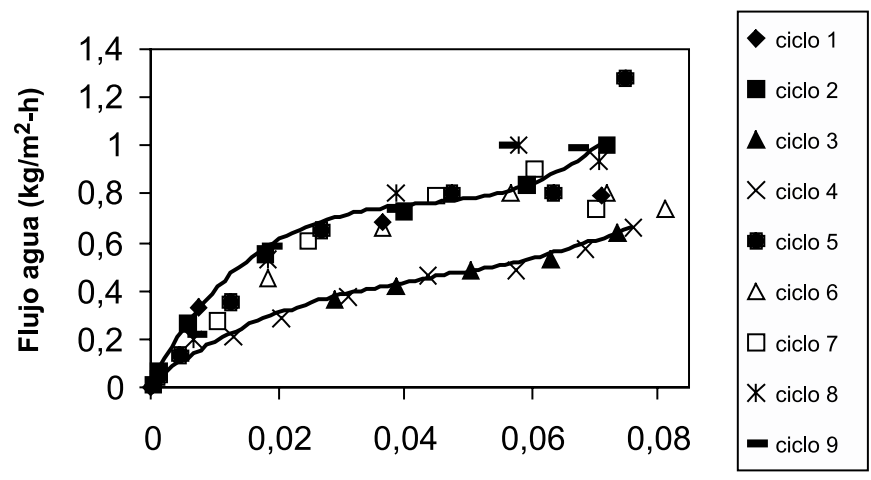

Fracción másica agua retenido

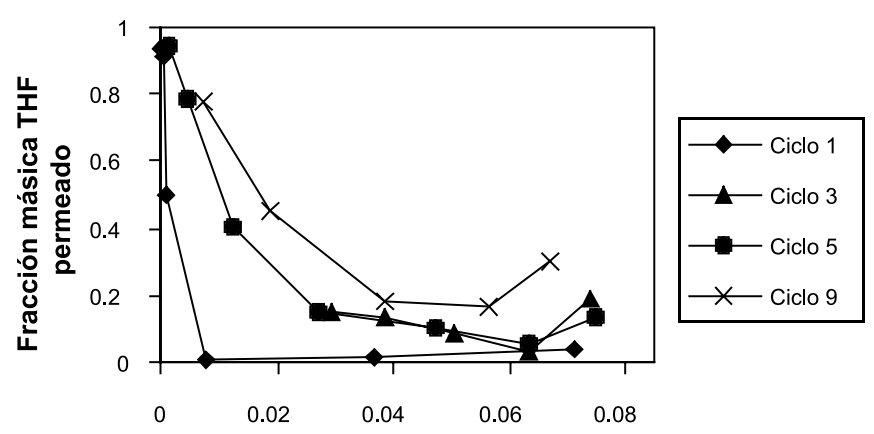

Fracción másica agua retenido

Figura 3. Flujo de agua y selectividad para la mezcla THF/agua, a 55 ${ }^{\circ} \mathrm{C}$, en nueve ciclos de operación. 
En cada nuevo ciclo se repone la mezcla de alimentación con una concentración inicial de agua de 7,9\% en peso. A partir de los transcursos concentración-tiempo, se obtiene el flujo de agua, representado en la figura 3(a). La selectividad expresada como fracción de THF en el permeado, se representa en la figura 3(b). Los ciclos 1 y 2 ofrecen resultados repetitivos en cuanto a flujo de agua y la selectividad de la separación. En los ciclos 1 y 2 la concentración de THF en el permeado es inferior al $1 \%$ en peso. Únicamente cuando la concentración de agua en el retenido es inferior a $0,05 \%$, el permeado se enriquece en THF.

Sin embargo en los ciclos 3 y 4 se observa la disminución del flujo de agua y de la selectividad. En esta situación se aplicó un procedimiento de limpieza que consistió en la realización de un ciclo de lavado y permeación con agua. Tras el procedimiento, en el ciclo 5 y consecutivos el flujo de agua volvió a los valores de los ciclos 1 y 2, tal como se observa en la figura 3(a). Sin embargo no ocurre lo mismo con la selectividad de la membrana, ya que se observa un aumento de la concentración de THF en el permeado, superando el $10 \%$ en la mayoría de las muestras. Estos resultados parecen indicar que la membrana puede haber modificado su estructura porosa debido al contacto con el agua. Las membranas utilizadas, comercializadas por Smart Chemicals, no vienen acompañadas de un procedimiento de limpieza y mantenimiento. Únicamente se conocen su extrema sensibilidad a $\mathrm{pH}$ inferior a 6, parámetro que no fue controlado en las operaciones de limpieza llevadas a cabo. En la literatura se encuentran referencias a ensayos de pervaporación con agua con membranas de tipo zeolita $\mathrm{NaA}(2,11)$ que sin embargo no hacen referencia a comportamientos inestables. El comportamiento inestable frente al agua de membranas cerámicas de pervaporación con un alto contenido en sílice ha sido referenciado por Asaeda et al. (12).

En la figura 4 se observa que el flujo de agua depende del gradiente de presión parcial de agua entre las dos caras de la membrana de pervaporación, el cual se suele considerar como la fuerza impulsora del transporte de agua a través de la membrana. En el caso de la mezcla THF/agua los datos obtenidos a $45^{\circ} \mathrm{C}$ y $55^{\circ} \mathrm{C}$ pueden adaptarse a una relación lineal, que sin embargo no parece poder aplicarse a los datos obtenidos a $65^{\circ} \mathrm{C}$. Para la mezcla acetona/agua la dependencia del flujo de agua con el gradiente de presión parciales similar que para el sistema THF/agua. En la bibliografía se recogen ambos tipos de dependencia del flujo con el gradiente de presión parcial, como la proporcionalidad entre ambos observada en la deshidratación de alcoholes con membranas zeolita tipo A y con membranas de sílice amorfa $(3,11)$ o la dependencia de tipo exponencial $(11,12)$.

\section{AGRADECIMIENTOS}

Los autores agradecen a la CICYT (MEC), la financiación recibida para la realización del trabajo a través del proyecto PPQ2000-0240. Asimismo, se agradece a la empresa Aries Ingeniería y Sistemas, S.A. el suministro de los materiales aportados.

\section{BIBLIOGRAFÍA}

1. Y. Morigami, M. Kondo, J. Abe, H. Kita, K. Okamoto. “The first large-scale pervaporation plant using tubular-type module with zeolite NaA membrane". Sep. Purf. Technol. 25, 251-260 (2001).

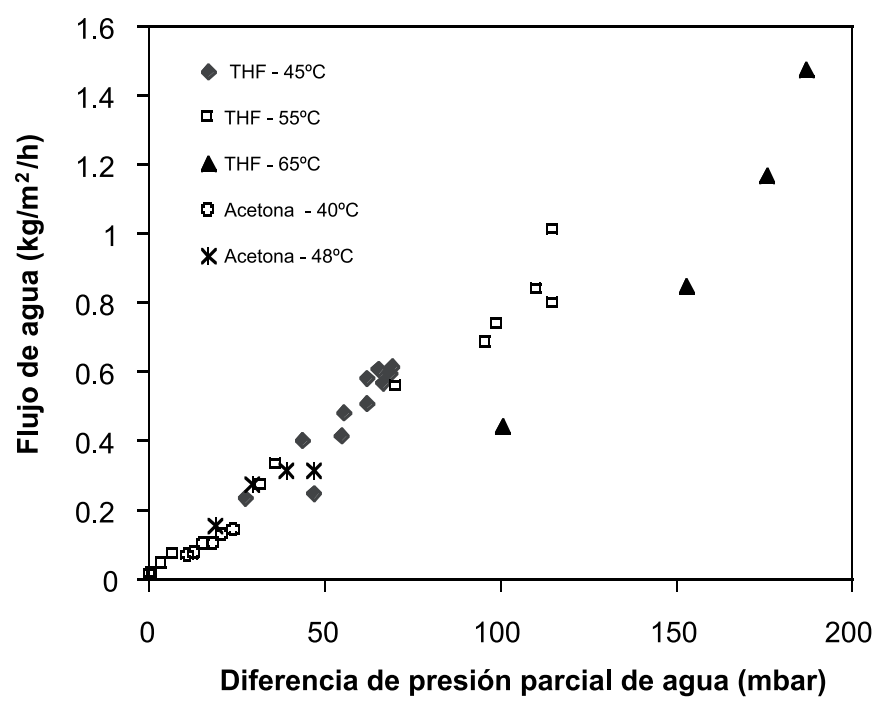

Figura 4. Dependencia del flujo de agua con el gradiente de presión parcial de agua

2. K. Okamoto, H. Kita, K. Horii, K. Tanaka, M. Kondo. “Zeolite NaA membrane: preparation, single-gas permeation, and pervaporation and vapor permeation of water/organic liquid mixtures". Ind. Eng. Chem. Res. 40, 163-175 (2001).

3. A.W. Verkerk, P. van Male, M.A.G. Vorstman, J.T.F. Keurentjes. “Description of Dehydration Performance of Amorphous Silica Pervaporation Membrane", J. Membr. Sci. 2001, 193, 227-238.

4. H.M. van Veen, Y.C. van Delft, C.W.R. Engelen, P.P.A.C. Pex. “Dewatering of Organics by Pervaporation with Silica Membranes", Sep. Purif. Technol. 2001, 22-23, 361-366.

5. R.H. Perry, D.W. Green, J.O. Maloney. "Manual del Ingeniero Químico". $7^{\mathrm{a}}$ edición. McGraw-Hill, Madrid, 2001.

6. T. Gallego-Lizón, E. Edwards, G. Lobuindo, L. Freitas dos Santos. “Dehydration of water/ $t$-butanol mixtures by pervaporation: comparative study of commercially available polymeric, microporous silica and zeolite membranes". J. Membr. Sci. 197, 309-319 (2002).

7. A.M. Urtiaga, E.D. Gorri, I. Ortiz. "Mass transfer modeling in the pervaporation of VOCs from diluted aqueous solutions". AIChEJ 48, 572-581 (2002).

8. A.M.Urtiaga, E.D.Gorri, J.K.Beasley, I. Ortiz. Mass Transfer analysis of the Pervaporative Separation of Chloroform from Aqueous Solutions in Hollow Fiber Devices". J. Membr. Sci. 1999, 156, 275-291.

9. A.M. Urtiaga, C. Casado, C. Aragoza, I. Ortiz, Dehydration of industrial ketonic effluents by pervaporation. Comparative behaviour of ceramic and polymeric membranes, Sep. Sci. Technol., 38 (2003) 3471.

10. S. Li, V.A. Tuan, R.D. Noble, J.L. Falconer. "Pervaporation of water/THF mixtures using zeolite membranes". Ind. Eng. Chem. Res. 2001, 40, 45774585 .

11. D. Shah, K. Kissick, A. Ghorpade, R. Hannah, D. Bhattacharyya. "Pervaporation of alcohol-water and dimethylformamide-water mixtures using hydrophilic zeolite NaA membranes: mechanisms and experimental results". J. Membr. Sci. 179, 185-205 (2000).

12. M. Asaeda, Y. Sakou, J. Yang, K. Shimasaki. "Stability and performance of porous silica-zirconia membranes for pervaporation of aqueous organic solutions". J. Membr. Sci. 209 (2002) 163-175.

Recibido: 01.2.03

Aceptado: 30.11 .03 